\title{
Ablagerung von gadoliniumhaltigen Kontrastmitteln im Gehirn nach mehrfacher Anwendung: Konsequenzen für den Einsatz der MRT bei Diagnosestellung und Verlaufsbeurteilung der Multiplen Sklerose?
}

\author{
Accumulation of Gadolinium-based Contrast Agents in the Brain Due to Repetitive Contrast-enhanced \\ MRI: Implications for the Use of MRI in the Diagnosis and Follow-up of Multiple Sclerosis Patients?
}

Autoren

Institute
C. Lukas ${ }^{1}$, R. Gold ${ }^{2}$, J. Fiehler ${ }^{3}$, S. Siemonsen ${ }^{3}$, J. F. Kleine ${ }^{4}$, F. Zipp ${ }^{5}$, B. Hemmer ${ }^{6}$, M. Mühlau

Die Institutsangaben sind am Ende des Beitrags gelistet
Schlüsselwörter

- Magnetresonanztomografie

- gadoliniumhaltige Kontrastmittel

- Gadoliniumablagerung

- Multiple Sklerose

\section{Keywords}

- magnetic resonance imaging

- gadolinium accumulation

- multiple sclerosis

- gadolinium-based contrast agents

\section{Zusammenfassung}

\section{$\nabla$}

Neue Studienergebnisse weisen auf einen $\mathrm{Zu}-$ sammenhang zwischen wiederholten kontrastmittel-unterstützen MRT-Untersuchungen und Ablagerung gadoliniumhaltiger Kontrastmittel im zentralen Nervensystem hin. Ein wesentlicher Faktor hierbei stellt möglicherweise die zum Einsatz kommende Kontrastmittelsubstanzklasse (linear bzw. makrozyklisch) dar. Über die letzten Jahrzehnte hat sich die MRT als ein unverzichtbarer Bestandteil in der Diagnostik, aber auch in der Verlaufsbeurteilung unterschiedlicher Erkrankungen etabliert. Dies gilt im besonderen Maße für chronische Erkrankungen, wie die Multiple Sklerose. Wiederholte MRT-Untersuchungen spielen hier insbesondere durch die zunehmenden Therapieoptionen eine wesentliche Rolle im Verlauf der Erkrankung. Im vorliegenden Artikel werden die derzeit vorhandenen Studienergebnisse über die Ablagerung gadoliniumhaltiger Kontrastmittel zusammengefasst und im Hinblick auf mögliche Konsequenzen für den Einsatz der MRT im Rahmen der Diagnose und insbesondere der Verlaufsbeurteilung der Multiplen Sklerose diskutiert.

\section{Bibliografie}

DOI http://dx.doi.org/

10.1055/s-0042-102953

Online-Publikation: 9.5.2016

Akt Neurol 2016; 43: 237-241

(c) Georg Thieme Verlag KG

Stuttgart · New York

ISSN 0302-4350

Korrespondenzadresse

Prof. Dr. med. Carsten Lukas

Institut für Diagnostische und Interventionelle Radiologie

St. Josef-Hospital

Ruhr-University Bochum

Gudrunstraße 56

44791 Bochum

carsten.lukas@rub.de

\section{Einleitung}

Bedingt durch neueste Studienergebnisse im Hinblick auf eine mögliche Akkumulation gadoliniumhaltiger Kontrastmittel (GBCA; gadolinium based contrast agent) im zentralen Nervensystem muss der generelle Einsatz dieser Kontrastmittel im Rahmen einer MRT-Untersuchung erneut kritisch hinterfragt werden. Die amerikanische Food and Drug Administration (FDA) hat in diesem Zusammenhang Ende Juli eine Sondermeldung herausgegeben, in der sie auf eine mögliche GBCA-Akkumulation bereits nach 4-maliger Anwendung aufmerksam macht (http://www.fda. gov/Drugs/DrugSafety/ucm455386.htm).

\section{Abstract \\ $\nabla$}

Recent studies have pointed towards a relationship between repetitive contrast- enhanced magnetic resonance imaging (MRI) and accumulation of gadolinium-based contrast agents. This relationship seems to be dependent on the subclass of contrast agents (linear vs. cyclic) used. Over the past decades, MRI has become one of the most valuable tools in the diagnosis and follow-up of a wide spectrum of disease entities. This holds true especially for chronic diseases such as multiple sclerosis. Given current strategies to establish MRI in treatment decision pathways due to the availability of more potential treatment options, repetitive MRI is frequently performed during the disease course of MS. In this article, we review currently available studies focusing on the accumulation of gadoliniumbased contrast agents. Furthermore, consequences that may arise in the context of MR imaging in MS patients are discussed. 
Verabreichung von GBCA magnetresonanztomografische Veränderungen detektieren lassen, welche auf eine vermehrte GBCA Ablagerung hindeuten. Jüngste Postmortem-Studien bestätigten diese Vermutung mittels direkter Bestimmung des Gadoliniums in verschiedenen Hirnregionen. Diese Veränderungen lassen sich jedoch nicht bei allen Patienten nachweisen. Ferner existieren bislang noch keine Hinweise auf gesundheitliche Risiken.

\section{Neue Beobachtungen zur Ablagerung gadoliniumhaltiger Kontrastmittel \\ $\nabla$}

GBCA galten lange Zeit als unbedenklich, da hier Gadolinium, ein toxisches paramagnetisches Schwermetall aus der Elementgruppe der seltenen Erden, nicht frei, sondern als Chelatkomplex vorliegt. Entsprechend der Chelat-Komplex-Struktur wird zwischen linearen und (makro)zyklischen GBCA unterschieden. Seit der Zulassung der ersten GBCA Ende der 80er Jahre wird die Zahl der Patientenuntersuchungen auf mehr als 100 Millionen geschätzt [1]. Schwerwiegende Nebenwirkungen werden in einer Größenordnung von 0,03\% angegeben, was das günstige Nebenwirkungsprofil belegt. Mit der Entdeckung der nephrogenen systemischen Fibrose (NFS) wurde erstmalig eine mögliche Gadoliniumablagerung im menschlichen Organismus mit einer seltenen, aber gravierenden Multiorgan-Erkrankung in Verbindung gebracht [2]. Dies gab Anlass zu Anwendungsbeschränkungen bei Niereninsuffizienz, da diese die Ausscheidung der GBCA verzögert und somit eine höhere Gefahr der Akkumulation im Organismus besteht. 2013 wurde in einer zunächst online veröffentlichten Arbeit von Kanda et al. erstmalig der Verdacht auf einen Zusammenhang zwischen häufigen GBCA-Gaben und Signalveränderungen im Nucleus dentatus und Globus pallidus (○ Abb. 1) geäußert, der wenig später von einer weiteren Arbeitsgruppe bestätigt wurde [3,4]. Dabei zeigte sich in den o.g. Hirnarealen eine signifikante positive Korrelation zwischen der kumulativen GBCA-Dosis und dem nativen T1-gewichteten Signal [4].

Grundsätzlich sollte darauf hingewiesen werden, dass eine Signalerhöhung in zentralnervösen Strukturen in einem nativen T1-gewichteten Bild auch andere Ursachen haben kann [5] und somit nicht zwingend auf vorausgegangenen GBCA-Applikationen beruht. V.a. in den zuvor genannten Studien ohne begleitende histopathologische Untersuchungen kann nicht zweifelsfrei ausgeschlossen werden, dass es sich bei den beschriebenen Fällen tatsächlich um GBCA-Ablagerungen handelt. Zwei unabhängige Arbeitsgruppen veröffentlichten jedoch nun erste post-mortem Ergebnisse [6,7], die u.a. mittels Plasma-Massenspektroskopie signifikant höhere Gadolinium-Konzentrationen im Hirngewebe (MW: 0,25ug/g Hirngewebe) bei Patienten mit vorausgegangenen seriellen GBCA-Gaben im Rahmen von MRT-Untersuchungen $(n=5)$ belegen [6]. Bei keinem der Patienten lagen Hinweise auf eine Nierenfunktionsstörung vor. Die Mehrzahl der Patienten hatte lineare GBCA erhalten. Die Konzentrationen waren insbesondere im Nucleus dentatus und Globus pallidus signifikant höher als in den übrigen untersuchten ZNS-Regionen [6], es fanden sich jedoch auch in weiteren Hirnarealen u. a. frontal sowohl im Kortex als auch im Marklager, im Pons sowie in der weißen Substanz des Kleinhirns Gadlinium-Konzentrationen, die deutlich über den Werten nicht exponierter Kontrollpersonen lagen [7]. Somit muss angenommen werden, dass sich GBCA in gewissen Grenzen auch bei intakter Nierenfunktion ablagern. Annähernd $1 / 3$ des nachweislichen Gadoliniums wurde im neurona-
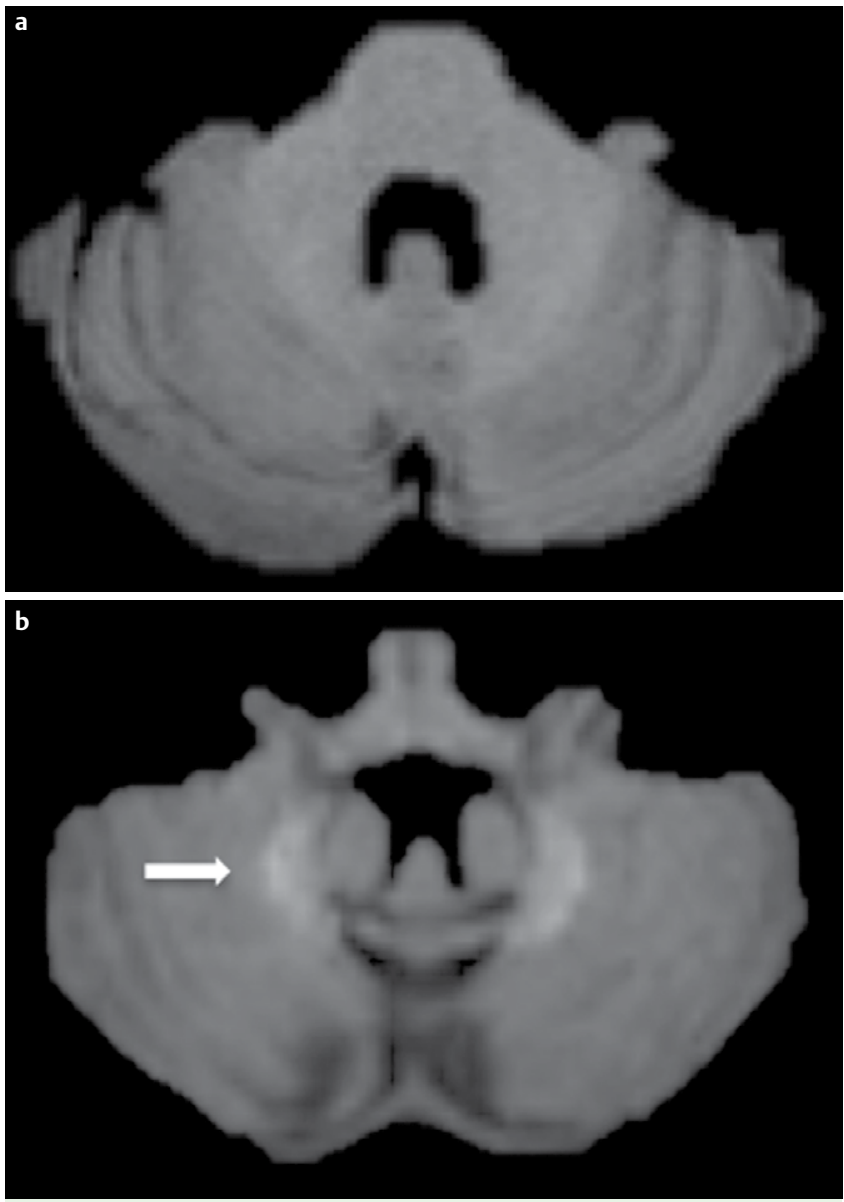

Abb. 1 Hyperintenses Signalverhalten des Nucleus dentatus a unauffälliges Signalverhalten des Nucleus dentatus in einem nativen T1-gewichteten Bild. b gesteigertes (hyperintenses) Signal des Nucleus dentatus (Pfeil) in einem nativen T1-gewichteten Bild bei einem MS Patienten mit mehrfachen kontrastmittelunterstützten MRT-Untersuchungen in den vergangenen Jahren.

len Interstitium nachgewiesen, was auf eine Passage der BlutHirn-Schranke (BHS) hinweist [7]. Die genauen Mechanismen, wie und in welcher Form Gadolinium die intakte BHS passiert wird, sind nicht geklärt.

Erste Ergebnisse lassen allerdings vermuten, dass zwischen den derzeit verfügbaren Substanzklassen Unterschiede in dieser Hinsicht existieren [8-11]. Retrospektive Analysen an $100 \mathrm{~Pa}-$ tienten mit repetitiver GBCA-Applikation (GadopentetateDimeglumine, linear, $n=50$; Gadoterate Meglumine, zyklisch, $\mathrm{n}=50$ ) lassen vermuten, dass makrozyklische GBCA ein geringeres Risiko bergen, da nur nach Applikation der linearen, nicht aber nach Applikation der zyklischen Form signifikante Signalerhöhungen im Nucleus dentatus nachweisbar waren [8]. $\mathrm{Zu}$ ähnlichen Ergebnissen kommen weitere Studien mit kleineren Fallzahlen [10] sowie tierexperimentelle Untersuchungen an Ratten [9]. Ferner konnte in einer kürzlich veröffentlichten Studie auch für das makrozyklische Gadobutrol keine signifikanten Signalerhöhungen in den relevanten Kerngebieten nach repetitiven Applikationen nachgewiesen werden [11]. Insgesamt scheint daher das Risikoprofil hinsichtlich der Akkumulation von Gadolinium bei makrozyklischen GBCA, analog zum Risiko einer NFS, günstiger auszufallen, was u.a. durch ein geringeres Risiko der Freisetzung von Gadoliniumionen begründet ist [12]. Eine abschließende Beurteilung erscheint jedoch auf Grund ers- 
ter kontroverser Ergebnisse (s.u.) noch nicht möglich [13]. Unklar ist ferner ob innerhalb der Gruppe der linearen GBCAs Unterschiede im Hinblick auf die Entwicklung von Signalauffälligkeiten existieren $[14,15]$.

\section{Bedeutung der Kontrastmittelgabe in der MR-Diagnostik der Multiplen Sklerose \\ $\nabla$}

Auch bei Patienten mit Multipler Sklerose (MS) wurden in der Vergangenheit Erhöhungen des T1-gewichteten Signals in der tiefen grauen Substanz u.a. im Nucleus dentatus $[16,17]$ oder in den Läsionen selbst [18] beschrieben. Diese Signalerhöhungen waren ausgeprägter bei sekundär chronisch progredienten Verläufen und zeigten eine enge Korrelation mit dem Behinderungsgrad. Als mögliche Ursachen wurden damals v.a. krankheitsassoziierte Faktoren wie eine abnormale Eisenablagerung im Rahmen neurodegenerativer Prozesse, reaktivierte eisenbeladene Mikroglia, paramagnetische freie Radikale oder die Remyelinisierung diskutiert [19-26]. Diese Ergebnisse müssen nun in einem neuen Licht betrachtet werden, da es nahe liegt, dass schwerer betroffene Patienten auch eine höhere kumulative GBCA-Dosis erhalten haben und erste Studien vorliegen, die eine solche Korrelation auch bei MS-Patienten nachwiesen [3,13]. Es bleibt zu klären, ob die bei der MS beschriebenen Signalintensitätserhöhungen ausschließlich ein Epiphänomen im Rahmen mehrerer kontrastmittelunterstützter MR Untersuchungen darstellen oder zumindest teilweise auch krankheitsspezifischer Natur sind $[3,13]$. Stojanov und Mitarbeiter untersuchten in einer kürzlich erschienenen Arbeit die Signalintensitäten im Nucleus dentatus und im Globus pallidus bei MS-Patienten $(n=58)$ mit schubförmigem Verlauf und glichen diese mit Häufigkeit und Zeitintervallen vorausgegangener KM-Untersuchungen ab [13]. Alle Patienten hatten in der Vergangenheit Gadubutrol, ein makrozyklisches GBCA, erhalten. Die Ergebnisse zeigen im Gegensatz zu der Arbeit von Radbruch und Mitarbeitern [11] eine abnormale Änderung der Signalintensität im Nucleus dentatus, die um so höher war, je kürzer die Frequenz zwischen den einzelnen Applikationen ausfiel. Methodische Unterschiede, welche nicht nur auf dem eingeschlossenen Patientenkollektiv beruhen (MS Patienten vs. Patienten mit Hirntumoren) erschweren einen direkten Vergleich zwischen beiden Studien. Unklar bleibt insbesondere, ob längere Zeitintervalle zwischen den Applikationen eine mögliche Akkumulation minimieren [14].

Der Einsatz von GBCAs im Rahmen der MRT-Untersuchung bei MS-Patienten hat sich in den letzten Jahren als ein fester Bestandteil in der Diagnosestellung, aber auch in Hinblick auf Verlaufsuntersuchungen etabliert. Die Anreicherung von asymptomatischen Läsionen stellt eine wesentliche Voraussetzung zur Erfüllung der zeitlichen Dissemination (DIT, dissemination in time) dar [27]. Ein Verzicht von Kontrastmittel könnte hier zu einer verzögerten Diagnosestellung, und damit verbunden, zur verzögerten Initiierung einer effektiven Behandlung führen. Ferner können im Rahmen der Erstuntersuchung durch den Einsatz von Kontrastmittel wichtige Zusatzinformationen im Hinblick auf mögliche Differenzialdiagnosen (z.B. die leptomeningeale Anreicherung bei Neurosarkoidose oder Borreliose) gewonnen werden. Der Einsatz im Rahmen der Erstdiagnostik sollte daher nicht hinterfragt werden. Deutlich schwerer fällt, den Nutzen von kontrastunterstützten MRT-Untersuchungen im Verlauf der Erkrankung zu belegen. Muss jeder Schub mittels MRT abgeklärt bzw. bestätigt werden? Und vor allem müssen alle Verlaufsuntersuchungen zwingend mit KM durchgeführt werden?

\section{Ist die Kontrastmittelgabe bei der Verlaufsbeurtei- lung der Multiplen Sklerose verzichtbar?}

Es besteht kein Zweifel, dass die MRT derzeit das sensitivste paraklinische Verfahren darstellt, um die Krankheitsaktivität der MS abzubilden. Störungen der BHS, wie sie im Rahmen aktiver MS Läsionen vorkommen, ermöglichen einen parazellulären Transport des GBCAs in das Interstitium, was sich in T1-gewichteten Sequenzen signalreich (hyperintens) vom umgebenen Hirnparenchym abgrenzt. Unbehandelt persistiert die Kontrastmittelanreicherung einer aktiven MS-Läsion in der Regel ca. 2-6 Wochen lang [28], eine Behandlung mit hochdosiertem Methylprednisiolon führt zu einer deutlichen zeitlichen Verkürzung der Kontrastmittelanreicherung [29,30]. Die Anzahl kontrastmittelaufnehmender Läsionen steigt unmittelbar vor bzw. während eines klinischen Schubereignisses an [31]. Die Vorhersagekraft kontrastmittelaufnehmender Läsionen in Bezug auf zukünftige Schubereignisse ist im kurzzeitigen Intervall allenfalls moderat [32].

In Hinblick auf eine „Schubsicherung“ bei etablierter Diagnose erscheint die MRT im klinischen Alltag generell nur selten notwendig und sollte Spezialfällen vorbehalten bleiben, z. B. zur Abgrenzung gegenüber funktionellen Beschwerden, die wahrscheinlich auch bei hochaktiven Patienten Schübe imitieren können [33]. Aber auch hierbei sollte bedacht werden, dass einige dieser Patienten schon im Vorfeld mit einer hochdosierten Methylprednisolonstoßtherapie behandelt wurden, welche bedingt durch den anti-inflammatorischen Effekt - das Zeitintervall einer Kontrastmittelanreicherung deutlich verkürzt, und somit den Nachweis KM-aufnehmender Läsionen vereiteln könnte [34]. Ferner zeigen jüngste Ergebnisse unter Einbeziehung hochauflösender 3D Sequenzen, dass sich ein nicht unerheblicher Anteil aktiver T2 Läsionen bereits vor Auftreten einer Kontrastmittelaktivität formiert, und somit nicht die Kontrastmittelaktivität per se den Beginn der im MRT sichtbaren Krankheitsaktivität definiert [35]. Zur Beurteilung des Verlaufs der MS mittels MRT ist zudem bedeutsam, dass eine Kontrastmittelaufnahme nicht nur bei der Formation neuer MS Läsionen auftritt, sondern auch bei bereits länger existierenden T2 Läsionen v.a. im Randbereich intermittierend aufflammen kann, wobei die prognostische Bedeutung dieser chronisch aktiven Herde unklar ist. Durch den Verzicht auf eine Kontrastmittelgabe im Rahmen der MRT-Verlaufsuntersuchung besteht somit ein gewisses, jedoch aller Wahrscheinlichkeit nach durchaus überschaubares Risiko, die aktuelle Krankheitsaktivität zu unterschätzen [36]. Vergleichsstudien zeigen jedoch, dass die Aussagekraft in Hinblick auf eine Behandlungseffektivität zwischen beiden „Aktivitätsmarkern“ (aktive Läsionen auf T1 post KM bzw. auf T2w Bildern) annähernd gleich stark ist [37].

Im Rahmen von Verlaufsuntersuchungen bei gesicherter Diagnose sollte daher der Einsatz von Kontrastmittel kritisch hinterfragt werden. Dies gilt insbesondere bei Patienten mit einem klinisch-stabilen Erkrankungsverlauf, da hier die subklinische, mittels T2-gewichteten Sequenzen detektierbare Krankheitsaktivität hinreichende Informationen liefern sollte. Bislang gibt es jedoch noch keine validierte Studie, auf deren Grundlage eine Therapieänderung bei klinisch stabilem Verlauf alleinig auf- 
grund neuer Marklagerläsionen im MRT gerechtfertigt werden könnte [38]. Allerdings wurden erste Vorschläge zur Implementierung von MRT-Parametern in klinische Entscheidungspfade veröffentlicht, die dies mitunter vorsehen [39-42]. Relevante MRT-Parameter sind hierbei der Nachweis aktiver Läsionen im zeitlichen Verlauf, welche als KM anreichernde Läsionen oder sich vergrößernde bzw. neue aufgetretene T2 Läsionen definiert werden [43]. Besagte Vorschläge basieren auf mehrfaktoriellen Strategien, bei denen neben MRT-Befunden auch klinische Parameter einbezogen werden $[41,42,44]$. Z. B. basiert der Rio-Score auf Untersuchungen möglicher MRT-Indikatoren für ein Ansprechen der Behandlung mit INF- 3 . Hierbei konnte der Nachweis von $\geq 3$ aktiven Läsionen innerhalb einer MRT-Verlaufsuntersuchung unter Therapie als prognostisch ungünstiger Faktor in Hinblick auf ein Therapieansprechen identifiziert werden [45]. Neben den klinischen Faktoren Schubanzahl und Krankheitsprogression (gemessen als EDSS Verschlechterung) bezieht der Score auch die MRT-Aktivität, gemessen als Anzahl aktiver Läsionen ein [42]. Für jede der 3 Einflussgrößen wird auf Grundlage des Vorhandenseins bzw. des Fehlens ein Punktewert von 1 bzw. 0 vergeben. Eine maximale Punktzahl von 3 zeigt daher ein hohes Risiko im Hinblick auf ein Therapieversagen an.

Später zeigten Sormani et al. mit dem sog. modifizierten RioScore auf, dass eine Risikoabschätzung auch nur alleinig durch den Nachweis von T2w Veränderungen ebenbürtig ist [46]. In der praktischen Routine könnte daher die Krankheitsaktivität alleinig durch die auf den T2w Bildern vorhandenen Informationen abgeschätzt werden. Voraussetzung hierfür ist natürlich eine standardisierte Durchführung der Untersuchungen, damit der sichere Nachweis neuer Läsionen durch den Vergleich der MRT-Bilder im Zeitverlauf überhaupt möglich wird. Standardisierte Untersuchungsabläufe können ebenfalls zur Dosisreduktion der zum Einsatz kommenden GBCA beitragen [47]. Dies gilt insbesondere bei bestehender Notwendigkeit einer gleichzeitigen zerebralen und spinalen MRT im Verlauf der Erkrankung. Hierbei kann durch Modifikation der Untersuchungsprotokolle eine mehrzeitige Messung, z. B. Aufteilung der Untersuchungsregionen auf 2 unterschiedliche Tage und damit verbundener 2-maliger KM-Applikation vermieden werden. Nach wie vor sinnvoll erscheint jedoch weiterhin die Verwendung kontrastmittelunterstützter MRT zur Beurteilung hochaktiver Verlaufsformen, um kurzfristig die Wirksamkeit einer Therapieeskalation abzuschätzen. Ebenso sollte auf GBCA nicht verzichtet werden, wenn eine progressive multifokale Leukenzephalopathie vermutet wird oder deren Verlauf beurteilt werden soll [48].

\section{Fazit für die Praxis}

Zusammenfassend sollte aufgrund der neuesten Daten, die eine Gadolinium-Anreicherung nach mehrfacher GBCA-Gabe in bestimmten Hirnregionen belegen, die Gabe von GBCA im Rahmen der Diagnosestellung nicht hinterfragt werden, während sie für routinemäßige Verlaufsuntersuchungen bei klinisch stabilem Verlauf in der Regel verzichtbar ist. Damit sollte sich die Zahl wiederholter GBCA-Gaben erheblich einschränken lassen. Wenn diese dennoch unvermeidlich sind, sollten nach dem derzeitigen Kenntnisstand vorzugsweise zyklische GBCA zum Einsatz kommen, da diese den linearen GBCA als Kontrastmittel ebenbürtig sind, sich aber möglicherweise weniger ablagern $[8,49]$. Inwieweit innerhalb der Substanzklasse der makrozyklischen Kontrastmittel potentiell sicherheitsrelevante Unterschiede bestehen, sollte durch weitere Studien genauer untersucht werden. Offen bleibt, ob und wie die Patienten aufgeklärt werden müssen. Nach derzeitiger Rechtsprechung hat der Arzt ebenfalls über atypische und evtl. für den medizinischen Laien überraschende Risiken aufzuklären, wenn sie im Einzelfall das Leben des Patienten schwer belasten können und auch bei geringer Komplikationsrate für den Eingriff spezifisch sind. Da bislang jedoch keine Daten über tatsächliche gesundheitliche Schäden solcher Ablagerungen, trotz weltweit langjähriger GBCA-Anwendung bekannt sind, kann nach dem aktuellen Kenntnisstand eine zwingende Aufklärungspflicht nach Ansicht der Autoren zumindest bei der Anwendung makrozyklischer Kontrastmittel nicht abgeleitet werden, weil es sich um mögliche, jedoch bis dato nicht nachgewiesene oder gar quantifizierbare gesundheitliche Risiken handelt.

\section{Danksagung}

Die Arbeit wurde durch das vom Bundesministerium für Bildung und Forschung (BMBF) geförderten krankheitsbezogenen Kompetenznetz Multiple Sklerose (KKNMS) unterstützt.

\section{Interessenkonflikt}

$\nabla$

Carsten Lukas erhielt Honorare für Vorträge und Mitarbeit in Advisory Boards von Bayer Vital GmbH, Biogen Idec GmbH, Genzyme, Novartis Pharma GmbH, Sanofi Aventis Deutschland GmbH, und Teva $\mathrm{GmbH}$ und erhielt Forschungsunterstützung von Merck-Serono GmbH, Bayer Vital GmbH, Teva GmbH und vom Bundesministerium für Bildung und Forschung (BMBF, „Krankheitsbezogenes Kompetenznetz Multiple Sklerose (KKNMS)“, 01GI0914). Er hält eine W2-Stiftungsprofessur für MS Imaging gefördert durch die Novartis-Stiftung inne. Ralf Gold erhielt Honorare für Vorträge oder Beratung bzw. Unterstützung für Forschungsvorhaben von Baxter, Bayer Healthcare, Biogen, CLB Behring, Genzyme, Merck-Serono, Novartis, Talecris, Teva, Wyeth. Jens Fiehler erhielt Vortragshonorare von Siemens und Philips; Susanne Siemonsen gibt an, dass keine Interessenkonflikte bestehen; Justus F. Kleine gibt an, dass keine Interessenkonflikte bestehen. Frauke Zipp hat Beratungen und wissenschaftliche Vorträge übernommen für Sanofi-Aventis, TEVA, Merck-Serono, Biogen, Genzyme, Novartis. Bernhard Hemmer gibt an, dass keine Interessenkonflikte bestehen. Mark Mühlau erhielt Wissenschaftsförderung von der DFG, KKNMS, HertieStiftung, Novartis und Merck-Serono.

\section{Institute}

'Institut für Diagnostische und Interventionelle Radiologie, St. Josef-Hospital, Ruhr-Universität Bochum, Bochum

${ }^{2}$ Klinik für Neurologie, St. Josef Hospital, Universität Bochum, Bochum ${ }^{3}$ Neuroradiologie, University Medical Center Hamburg-Eppendorf, Hamburg

${ }^{4}$ Abteilung für Neuroradiologie, Technische Universität, München

${ }^{5}$ Klinik und Poliklinik für Neurologie, Universitätsmedizin Mainz, Johannes

Gutenberg-Universität Mainz, Mainz

${ }^{6}$ Abteilung für Neurologie, Technische Universität, München

\section{Literatur}

1 Kanal E, Tweedle MF. Residual or retained gadolinium: practical implications for radiologists and our patients. Radiology 2015; 275: 630-634

2 Grobner T. Gadolinium - a specific trigger for the development of nephrogenic fibrosing dermopathy and nephrogenic systemic fibrosis? Nephrol Dial Transplant 2006; 21: 1104-1108 
3 Errante Y, Cirimele V, Mallio CA et al. Progressive increase of T1 signal intensity of the dentate nucleus on unenhanced magnetic resonance images is associated with cumulative doses of intravenously administered gadodiamide in patients with normal renal function, suggesting dechelation. Invest Radiol 2014; 49: 685-690

4 Kanda T, Ishii K, Kawaguchi $\mathrm{H}$ et al. High signal intensity in the dentate nucleus and globus pallidus on unenhanced T1-weighted MR images: relationship with increasing cumulative dose of a gadolinium-based contrast material. Radiology 2014; 270: 834-841

5 Ginat DT, Meyers SP. Intracranial lesions with high signal intensity on T1-weighted MR images: differential diagnosis. Radiographic 2012; 32: 499-516

6 Kanda T, Fukusato T, Matsuda $M$ et al. Gadolinium-based contrast agent accumulates in the brain even in subjects without severe renal dysfunction: evaluation of autopsy brain specimens with inductively coupled plasma mass spectroscopy. Radiology 2015; 276: 228-232

7 McDonald RJ, McDonald JS, Kallmes DF et al. Intracranial gadolinium deposition after contrast-enhanced $\mathrm{mr}$ imaging. Radiology 2015; 275: 772-782

8 Radbruch A, Weberling LD, Kieslich PJ et al. Gadolinium retention in the dentate nucleus and globus pallidus is dependent on the class of contrast agent. Radiology 2015; 275: 783-791

9 Robert P, Lehericy Sp, Grand S et al. T1-weighted hypersignal in the deep cerebellar nuclei after repeated administrations of gadoliniumbased contrast agents in healthy rats: difference between linear and macrocyclic agents. Invest Radiol 2015; 50: 473-480

10 Kanda T, Osawa $\mathrm{M}$, Oba $\mathrm{H}$ et al. High signal intensity in dentate nucleus on unenhanced T1-weighted MR images: association with linear versus macrocyclic gadolinium chelate administration. Radiology 2015; 275: 803-809

11 Radbruch A, Weberling LD, Kieslich PJ et al. High-signal intensity in the dentate nucleus and globus pallidus on unenhanced T1-weighted images: evaluation of the macrocyclic gadolinium-based contrast agent gadobutrol. Invest Radiol 2015; 50: 805-810

12 Idee JM, Port M, Dencausse A et al. Involvement of gadolinium chelates in the mechanism of nephrogenic systemic fibrosis: an update. Radiol Clin North Am 2009; 47: 855-869 vii

13 Stojanov DA, Aracki-Trenkic A, Vojinovic S et al. Increasing signal intensity within the dentate nucleus and globus pallidus on unenhanced T1W magnetic resonance images in patients with relapsing-remitting multiple sclerosis: correlation with cumulative dose of a macrocyclic gadolinium-based contrast agent, gadobutrol. Eur Radiol 2016; 26: $807-815$

14 Weberling $L D$, Kieslich PJ, Kickingereder $P$ et al. Increased signal intensity in the dentate nucleus on unenhanced T1-weighted images after gadobenate dimeglumine administration. Invest Radiol 2015; 50: 743-748

15 Ramalho J, Castillo M, AlObaidy $M$ et al. High signal intensity in globus pallidus and dentate nucleus on unenhanced T1-weighted MR images: evaluation of two linear gadolinium-based contrast agents. Radiology 2015; 276: 836-844

16 Roccatagliata L, Vuolo L, Bonzano L et al. Multiple sclerosis: hyperintense dentate nucleus on unenhanced T1-weighted MR images is associated with the secondary progressive subtype. Radiology 2009; 251: 503-510

17 Absinta M, Rocca MA, Filippi M. Dentate nucleus T1 hyperintensity in multiple sclerosis. AJNR 2011; 32: E120-E121

18 Janardhan V, Suri S, Bakshi R. Multiple sclerosis: hyperintense lesions in the brain on nonenhanced T1-weighted MR images evidenced as areas of T1 shortening 1. Radiology 2007; 244: 823-831

19 Brass SD, Chen NK, Mulkern RV et al. Magnetic resonance imaging of iron deposition in neurological disorders. Top Magn Reson Imaging 2006; 17: 31-40

20 Drayer B, Burger P, Hurwitz B et al. Reduced signal intensity on MR images of thalamus and putamen in multiple sclerosis: increased iron content? AJR 1987; 149: 357-363

21 Gutteridge JM. Iron and oxygen radicals in brain. Ann Neurol 1992; 32 (Suppl): S16-S21

22 LeVine SM. Iron deposits in multiple sclerosis and Alzheimer's disease brains. Brain Res 1997; 760: 298-303

23 Powell T, Sussman JG, Davies-Jones GA. MR imaging in acute multiple sclerosis: ringlike appearance in plaques suggesting the presence of paramagnetic free radicals. AJNR 1992; 13: 1544-1546

24 Shan DE, Pan HC, Ho DM et al. Presence of activated microglia in a high-signal lesion on T1-weighted MR images: a biopsy sample reexamined. AJNR 2007; 28: 602
25 Terada H, Barkovich AJ, Edwards MS et al. Evolution of high-intensity basal ganglia lesions on T1-weighted MR in neurofibromatosis type 1. AJNR 1996; 17: 755-760

26 Zhou F, Shiroishi M, Gong $H$ et al. Multiple sclerosis: hyperintense lesions in the brain on T1-weighted MR images assessed by diffusion tensor imaging. Journal of magnetic resonance imaging: JMRI 2010; 31: 789-795

27 Polman CH, Reingold SC, Banwell B et al. Diagnostic criteria for multiple sclerosis: 2010 revisions to the McDonald criteria. Ann Neurol 2011; 69: 292-302

28 Vigeveno RM, Wiebenga OT, Wattjes MP et al. Shifting imaging targets in multiple sclerosis: from inflammation to neurodegeneration. Journal of magnetic resonance imaging: JMRI 2012; 36: 1-19

29 Barkhof F, Hommes OR, Scheltens P et al. Quantitative MRI changes in gadolinium-DTPA enhancement after high-dose intravenous methylprednisolone in multiple sclerosis. Neurology 1991; 41: 1219-1222

30 Burnham JA, Wright RR, Dreisbach J et al. The effect of high-dose steroids on MRI gadolinium enhancement in acute demyelinating lesions. Neurology 1991; 41: 1349-1354

31 Filippi M, Rocca MA. MR imaging of multiple sclerosis. Radiology 2011; 259: 659-681

32 Kappos L, Moeri D, Radue EW et al. Predictive value of gadoliniumenhanced magnetic resonance imaging for relapse rate and changes in disability or impairment in multiple sclerosis: a meta-analysis. Gadolinium MRI Meta-analysis Group. Lancet 1999; 353: 964-969

33 Fox RJ, Cree BA, De Seze J et al. MS disease activity in RESTORE: a randomized 24-week natalizumab treatment interruption study. Neurology 2014; 82: 1491-1498

34 Cotton F, Weiner HL, Jolesz FA et al. MRI contrast uptake in new lesions in relapsing-remitting MS followed at weekly intervals. Neurology 2003; 60: 640-646

35 Guttmann CR, Rousset $M$, Roch JA et al. Multiple sclerosis lesion formation and early evolution revisited: A weekly high-resolution magnetic resonance imaging study. Mult Scler 2015, Online First doi:10.1177/1352458515600247

36 Miller DH, Barkhof F, Nauta JJ. Gadolinium enhancement increases the sensitivity of MRI in detecting disease activity in multiple sclerosis. Brain 1993; 116: 1077-1094

37 Bonzano L, Roccatagliata L, Mancardi GL et al. Gadolinium-enhancing or active T2 magnetic resonance imaging lesions in multiple sclerosis clinical trials? Mult Scler 2009; 15: 1043-1047

38 Filippi M, Rocca MA, Arnold DL et al. Use of imaging in multiple sclerosis. In: European Handbook of Neurological Management. Gilhus NE, Brainin MM. ed. Blackwell Publishing; 2011: 35-51

39 Freedman MS, Patry DG, Grand'Maison F et al. Treatment optimization in multiple sclerosis. Can J Neurol Sci 2004; 31: 157-168

40 Freedman MS, Selchen D, Arnold DL et al. Treatment optimization in MS: Canadian MS Working Group updated recommendations. Can J Neurol Sci 2013; 40: 307-323

41 Stangel M, Penner IK, Kallmann BA et al. Multiple Sclerosis Decision Model (MSDM): Entwicklung eines Mehrfaktorenmodells zur Beurteilung des Therapie- und Krankheitsverlaufs bei schubförmiger Multipler Sklerose. Akt Neurol 2013; 40: 486-493

42 Rio J, Castillo J, Rovira A et al. Measures in the first year of therapy predict the response to interferon beta in MS. Mult Scler 2009; 15: 848-853

43 Dobson R, Rudick RA, Turner $B$ et al. Assessing treatment response to interferon-beta: is there a role for MRI? Neurology 2014; 82: 248-254

44 Grand'Maison F, Bhan V, Freedman MS et al. Utility of the Canadian Treatment Optimization Recommendations (TOR) in MS care. Can J Neurol Sci 2013; 40: 527-535

45 Rio J, Rovira A, Tintore $M$ et al. Relationship between MRI lesion activity and response to IFN-beta in relapsing-remitting multiple sclerosis patients. Mult Scler 2008; 14: 479-484

46 Sormani MP, Rio J, Tintore $M$ et al. Scoring treatment response in patients with relapsing multiple sclerosis. Mult Scler 2013; 19: 605-612

47 Simon JH, Li D, Traboulsee A et al. Standardized MR imaging protocol for multiple sclerosis: Consortium of MS Centers consensus guidelines. AJNR 2006; 27: 455-461

48 Wattjes MP, Barkhof F. Diagnosis of natalizumab-associated progressive multifocal leukoencephalopathy using MRI. Curr Opin Neurol 2014; 27: 260-270

49 Malayeri AA, Brooks KM, Bryant $H$ et al. National Institutes of Health Perspective on Reports of Gadolinium Deposition in the Brain. J Am Coll Radiol 2016; 13: 237-241 